\title{
The Colony Count Based on Image Processing Using Matlab and Photoshop
}

\author{
Xiaoming Chen, Shufang $\mathrm{Li}^{*}$, Zhijun Zhang and Jun Gao \\ TianJin Institute of Forestry and Pomology, Tianjin, China \\ ${ }^{*}$ Corresponding author
}

\begin{abstract}
Using two conventional software programs to do a counting method on the colony image from a normal photograph, and the comparison between the methods.
\end{abstract}

Keywords—colony counting; image processing; Matlab; Photoshop

\section{INTRODUCTION}

Although modern and automated advanced instruments can doing well of counting the colony count, the simple image processing and counting of the colony images, especially from ordinary digital cameras and mobile phone cameras, is still significant.

In this paper, we used two common software, Matlab and Photoshop, to experiment and compare three simple methods of colony counting with simple image processing.

\section{MATERIAL}

\section{A. Subject}

The colony is from Lactobacillus spp. and Saccharomyces cerevisiae by drop plate methods [1,2] in PDA culture mediums. The PDA plate culture medium at $25^{\circ} \mathrm{C}$ was cultured after $48 \mathrm{~h}$.

\section{B. Software}

Using three software, mathematic software as Matlab R2014a 32-bit; image processing software as Adobe Photoshop CC 2017 32-bit for colony counting

Results data was processed and statistics Microsoft Office EXCEL 2013 32-bit.

\section{Instrument}

Canon 450d digital camera for image taken.

\section{METHODS}

\section{A. Image Acquisition and Pretreatment}

For simplicity and convenience this experiment uses a Canon digital camera 450D to photograph the colonies which is formed by drop plate methods on a petri dish.

The camera using the attached $28-70 \mathrm{~mm}$ zoom lens during the shoot. The lens parameters are set to the focal length $=70$ $\mathrm{mm}$; Aperture $\mathrm{F}=5$.6.Camera body parameter are set to: sensitivity =200 ISO; Shutter speed $S=1 / 150$ s.camera manual mode to "Macro mode" and "Auto focus". All other parameters are camera default.

During shooting, the culture dish containing colonies was lined with black background and illuminated with natural light.

After the photo is exported from the camera, it is loaded into Adobe Photoshop. Using the "select" tool in the software, the image of the colony was intercepted to square as the side length of about $13.34 \mathrm{~cm}$, and the resolution was about 72ppi.The captured photos are stored in.jpg format. The original image is shown in figure I.
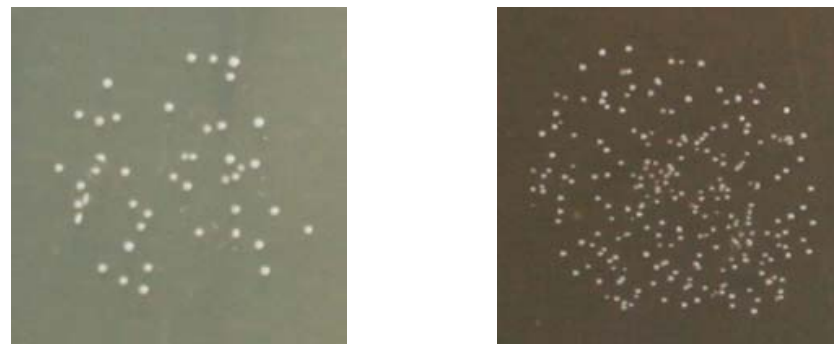

FIGURE I. TWO KINDS OF TYPECAL ORIGINAL IMAGE PROCESSED BY PHOTOSHOP

\section{B. Image Processing and Counting Methods Using Adobe Photoshop}

Select the "color range" option in the "select" menu bar in Photoshop. When the dialog box pops up, the cursor of the shape of the dropper is directly selected for any single colony sampling in the center of the image. When the dialog box pops up, the cursor in the shape of the "dropper" selects any single colony sample in the center of the image.

After sampling, click "ok" and the colony will be surrounded by a dotted line. At this point, click the "measurement record" tab in the "window" menu bar, and you will see the "measurement record" window. Records at this time click the interface "measure" button, you can record in "measurement" window displays the number of colonies surrounded by a dashed line (selected by the dotted line not colonies cannot be count and display). The operation diagram is shown in figure II. This method is abbreviated to M1. 


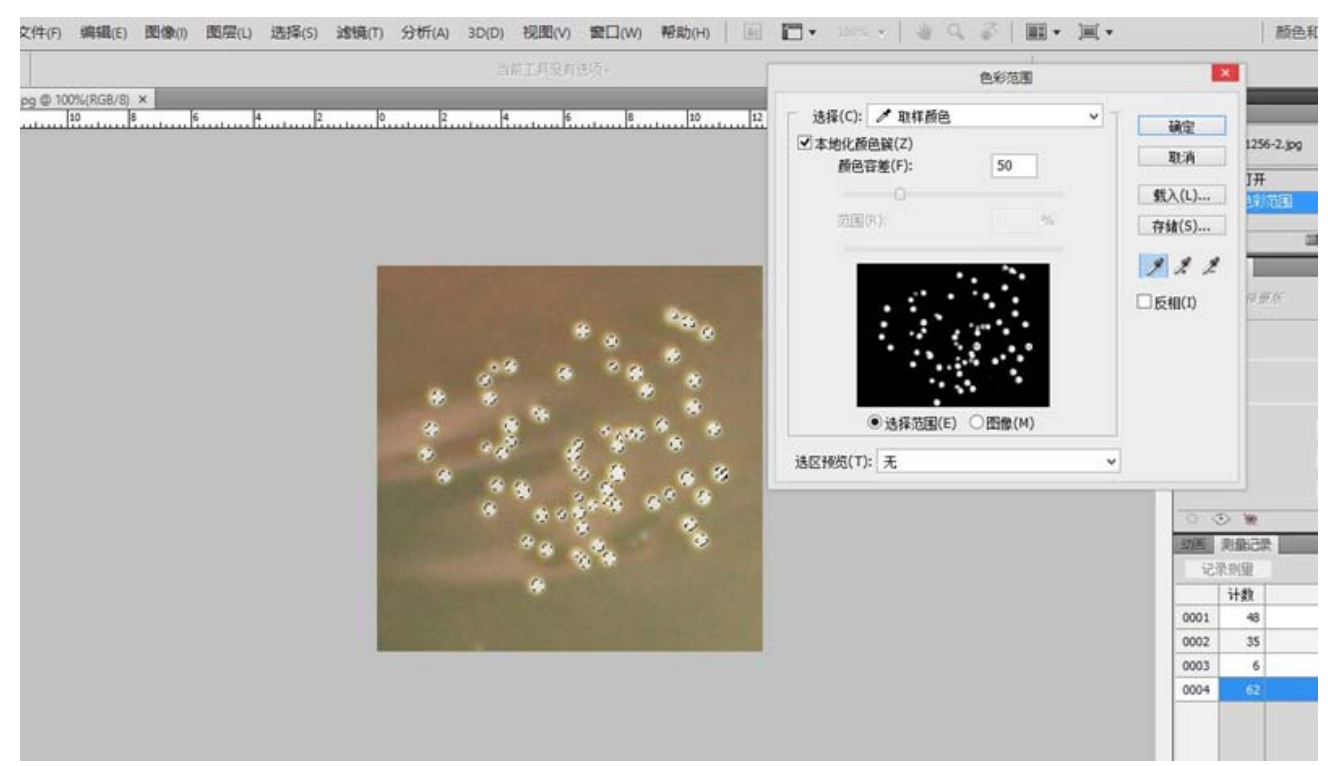

FIGURE II. SHOWS THE OPERATION SCHEMATIC DIAGRAM USING THE COLOR RANGE COUNT OF PHOTOSHOP

C. Another Method of Image Processing and Counting Using Adobe Photoshop

Using the "image" menu under the Photoshop menu, "adjust" the "black and white" button in the menu of the "adjust" submenu to transform the image into a black-andwhite image. Adjust the image threshold to the current half through the "threshold" menu in the same submenu. Select the "color range" option in the "select" menu bar in Photoshop. When the dialog box pops up, the cursor of the shape of the dropper is directly selected for any single colony sampling in the center of the image. When the dialog box pops up, the cursor in the shape of the "dropper" selects any single colony sample in the center of the image.

After sampling, click "ok" and the colony will be surrounded by a dotted line. At this point, click the "measurement record" TAB in the "window" menu bar, and you will see the "measurement record" window. Records at this time click the interface "measure" button, you can record in "measurement" window displays the number of colonies surrounded by a dashed line (selected by the dotted line not colonies cannot be count and display). The operation diagram is shown in figure III. This method is abbreviated to M2.

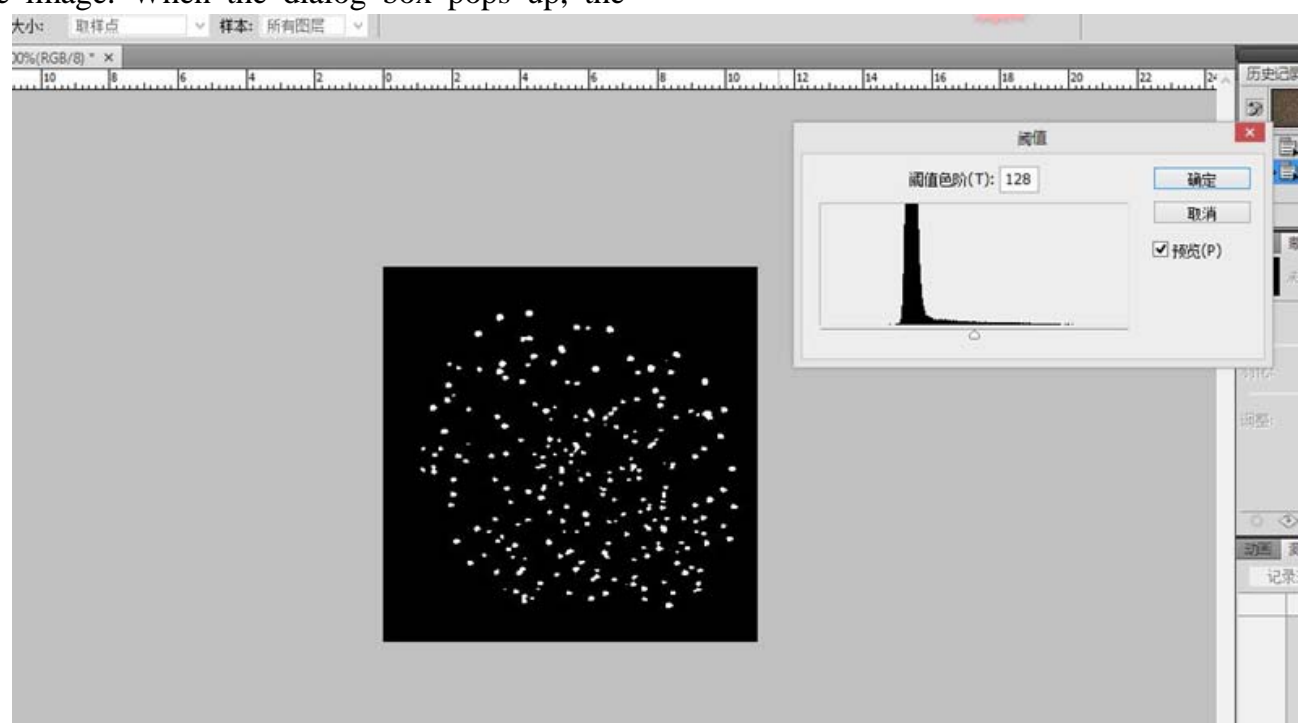

FIGURE III. USING PHOTOSHOP GRAYSCALE COLOR RANGE COUNTING OPERATION SCHEMATIC.

\section{Image Processing and Counting Methods Through Software Matlab}

The collected image import Matlab folders in the Matlab software, the image converted to black and white image banalization, the ash filtering, through top-hat transform and bottom hat transformations, Unicom area count, this method shorthand for M3[3,5]. 
The operation steps of the above method are as follows: enter the following five sets of program codes in the Matlab command input box:
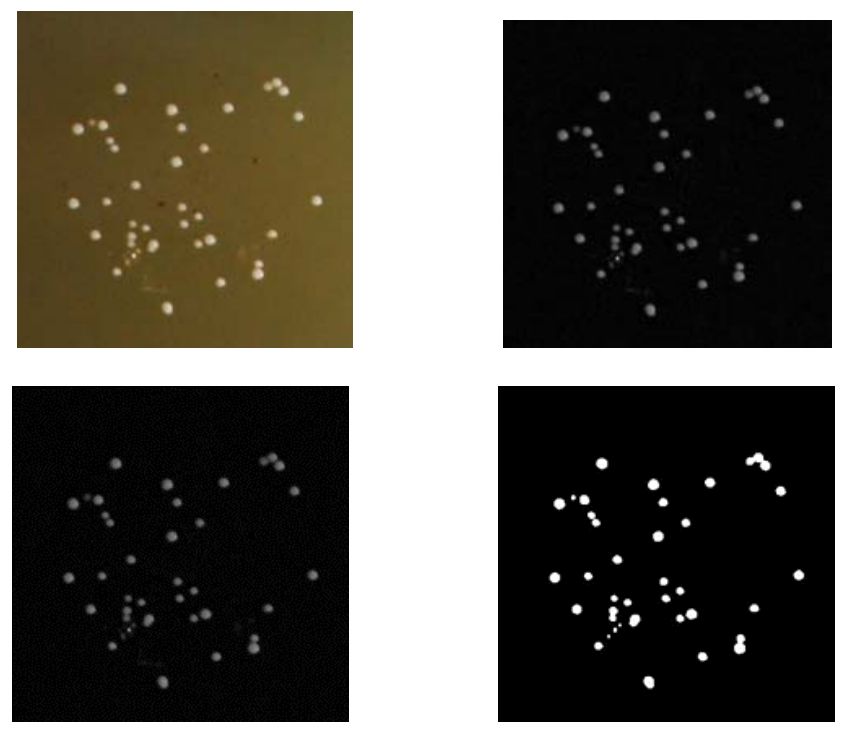

FIGURE IV. TWO KINDS OF ORIGINAL IMAGE(A) ORIGINAL IMAGE PARTS OF A FIGURE. (B) IMAGE BY TOP-HAT FILTERING CONVENT (C) GARY IMAGE BY 2-D MEDIAN FILTERING CONVENT (D) IMAGE BY BINARY CONVENT USING LEVEL THRESHOLD

- Import Image. The program code is as follows, The original image is shown in figure IV (A):
o clear
o filename='filename.jpg';
o imfinfo (filename);
o f=imread (filename);
o subplot (221),imshow (f);
o title ('Original image');

- Using Top-hat Filtering to correct uneven illumination or flash Spot. The program code is this, By Top-hat Filtering convert, the image is transformed into a form as shown in figure IV (B).

o se=strel ('disk',10);
o f1=imtophat (f,se);
o subplot (222), imshow (f1);
o title ('Image by imtophat filtering');

- Convert the Top-hat filtering image to grayscale image. The program code is this:
O $\quad$ A2 = rgb2gray (f1);
o imwrite (A2,'A2=gray.jpg');

- Using the 2-D median filtering to Remove Salt and Pepper Noise from Image. The program code is as follows, Removing Salt and Pepper Noise,the image is transformed into a form as shown in figure IV (D).
o $\quad$ A3 = medfilt2 (A2);
o subplot (223), imshow (A3);
o title ('Gary image by medfilt2 Filtering');

- Convert an Indexed Intensity Image to Binary Image Using Level Threshold. The program code is this, Removing Salt and Pepper Noise, the image is transformed into a form as shown in figure IV (D).

$$
\begin{array}{ll}
\text { o } & \text { Level = graythresh (A3); } \\
\text { o } & \text { imwrite (A3,'A3=medfilt2gray.jpg'); } \\
\text { o } & \text { A4 = im2bw (A3,level); } \\
\text { o } & \text { subplot (224), imshow (A4); } \\
\text { o } & \text { title ('Image by BW Filtering'); }
\end{array}
$$

- 6. Label Components Using 4-connected Objects, and print out the number of components. The program code is as follows, label Components, the software input result of colony counting by command window.

$$
\begin{array}{ll}
\text { o } & \text { imwrite (A4,'A4=bwmedfilt2gray.jpg'); } \\
\text { o } & {[1, n]=\text { bwlabel (A4,4); }} \\
\text { o } & \text { display (n); }
\end{array}
$$

\section{RESUlT}

Taking the results of the colony count of 10 pictures as an example, the results of the colony count produced by each image processing method are shown in figue $\mathrm{V}$. 


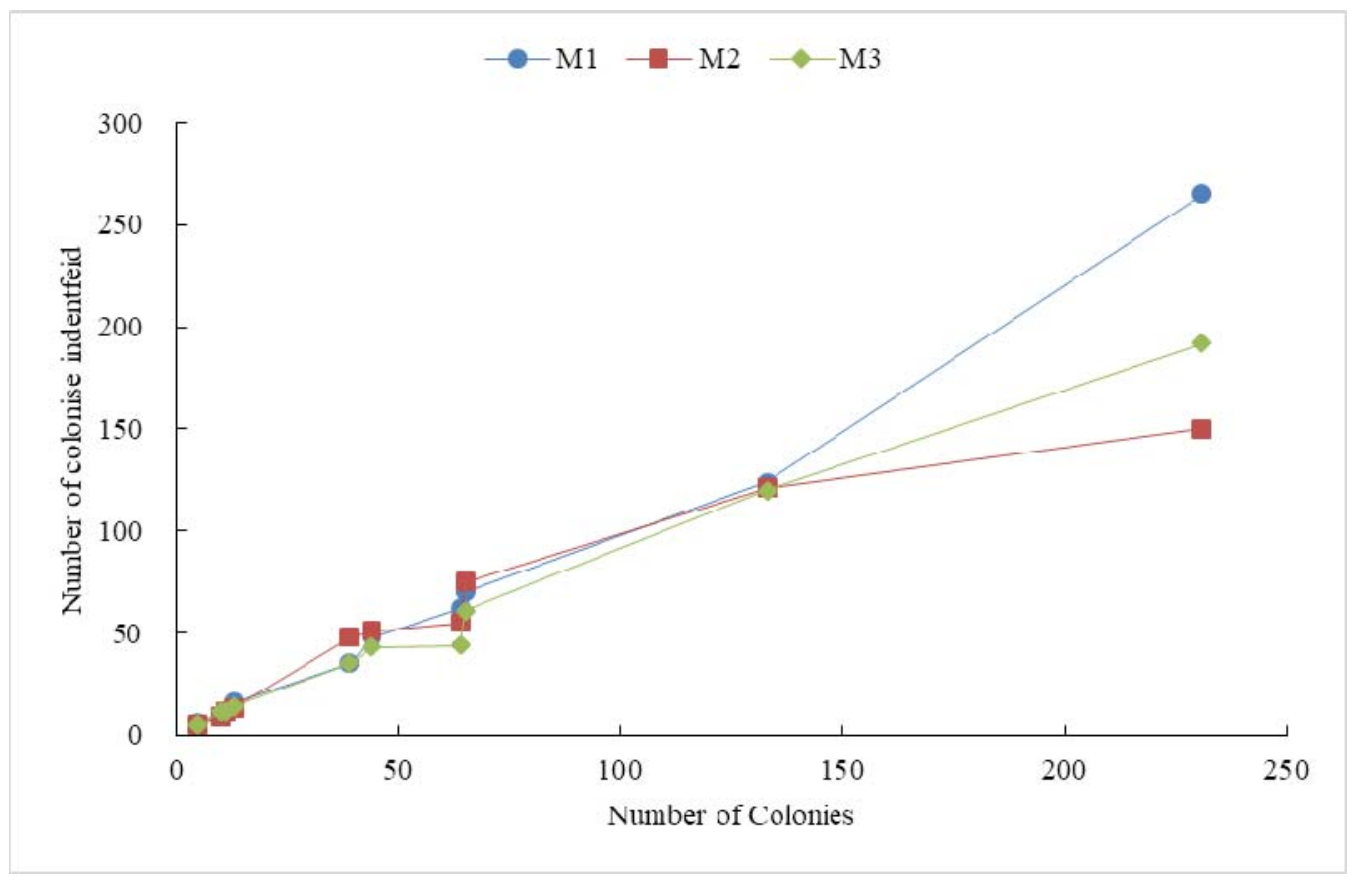

FIGURE V. RESULTS OF COLONY COUNT OF EACH METHOD

It can be seen that all three methods have good accuracy when the number of colonies is less than 50, and when the number of colonies is greater more than 100 , the recognition result of the method M1 is closer to the actual number of colonies.

\section{ACKNOWLEDGEMENT}

This research was financially supported by the National Public Welfare Wector (agriculture) research and special Foundation of China (201503142-09).

\section{REFERENCES}

[1] Hoben HJ, Somasegaran P. Comparison of the pour, spread, and drop plate methods for enumeration of Rhizobium spp. In inoculants made from presterilized peat [J]. Applied and Environmental Microbiology, 1982, 44(5): 1246-1247.

[2] Herigstad B, Hamlton M, Heersnk J. How to optimize the drop plate method for enumerating bacteria [J]. Journal Microbiology Method, 2001, 44 (2): 1212-1291.

[3] Marotz J. Effective object recognition for automated counting of colonies in Petri dishes (automated colony counting). Computer Methods and Programs in Biomedicine 66 (2001).

[4] Wu D Y, Wei P. Measuring The Condition of Parking Lot by Image Processing [J]. Journal of Beijing Institute of Technology, 1999, 8(3): 232-237.

[5] Hieu T, Marcel M, Rein V. Water snakes: Energy driven watershed segmentation [J], IEEE Transaction on Pattern Analysis and Machine Intelligence, 2003, 25(3): 330-342. 